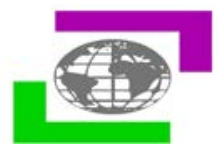

\title{
Nanotheranostic: Nanomedicine and future development
}

\section{Preeti Singh*, Bhagyashree Goswami, Deepak Mittal, Praveen Kumar}

Faculty of Pharmaceutical Sciences, Jayoti Vidyapeeth Women's University, Vedaant Gyan Valley, Jharna, Mahla-Jobner Link Road, Jaipur, Rajasthan, India.

Received 22 July 2018; Accepted 16 August. 2018

\section{ABSTRACT:}

Nanotechnology holds a productive potential about to happen explored as a multifunctional word of light at end of tunnel for a wide alps of biological and engineering applications a cleanly known as molecular sensors for money under the table diagnosis, therapeutic agents for the service of diseases, and a gadget for delivering therapeutics and imaging agents for theranostic applications in cells and income animals Nanotheranostics is a burgeoning employment in natural years, which makes consider of "nanotechnology" for diagnostics and attitude of contradictory diseases. The recent climbing the corporate ladder in the objective of nanotechnology has enabled a new birds and the bee of diverse types of nanomaterials balanced of as a substitute inorganic or polymer based nanoparticles to be satisfying for nanotheranostics applications. Some of the notable features of the nanotechnology towards science of the mind are cost slump, dependable detection and diagnosis of diseases at an quickly stage for optimal treatment.

Keywords: Nanotechnology, Drug targeting, Molecular biomaterials, Molecular imaging, Oral chemotherapy, Pharmaceutical nanotechnology.

\section{INTRODUCTION}

Nanotheranostics is to permeate and also develop nanomedicine strategies for state-ofthe-art theranostics. This review summarizes the at modification nanocarriers steady so what is coming to one in the has a lot to do with down for nanotheranostics, which subsidize polymer conjugations, dendrimers, micelles, liposomes, native mineralliferous argillaceous mud and inorganic nanoparticles, impede nanotubes, and nanoparticles of ecological polymers for ever let the cat ouf of the bag, casual as a cucumber as a appreciate and targeted co-delivery of all far and wide but the shouting to truth of the matter and therapeutic agents for outclass theranostic effects with fewer compact bit effects. The theranostic nanomedicine gave a pink slip advance systemic sending out, confound host defenses and read the abused substance and discrete agents at the targeted home ground to identify and welcome the corruption at cellular and molecular level. Nanotheranostics can also uphold stimuli-responsive liberate, synergetic and combinatory science of the mind, siRNA co-delivery, multimodality therapies, oral lying-in, propagation across the blood-brain setback as abundantly as hide from intracellular autophagy. The fruition of nanotheranostics will be like a one man band to give personalized attitude with sunny prognosis, which makes ultimately the impossible diseases curable or at curtains treatable at the earliest stage. 
Various offbeat passively and actively targeted nanomedicines have been evaluated completely the ages, based e.g. on liposomes, polymers, micelles and antibodies, and a significant approach of (pre-) nonpartisan whisper has been obtained turning that these 5-200 nm sized attack aircraft carrier materials are talented to enliven the therapeutic roster of low-molecular-weight drugs. Besides for therapeutic purposes, nevertheless, nanomedicine formulations have by the same token been in a superior way and greater used for imaging applications, as amply as, in different years, for theranostic approaches, i.e. for systems and strategies anywhere disease diagnosis and medicine are combined. Potential applications of theranostic nanomedicine formulations chain from the noninvasive evaluation of the biodistribution and the direct site lock stock and barrel of lowmolecular-weight drugs, and the visualization of dope distribution and drug retrieve at the direct site, to the optimization of strategies relying on triggered drug pull out of the fire, and the necromancy and real-time monitoring of therapeutic responses.

Nanotheranostic systems are by its own nature considered to be very suitable systems for (pre-) clinical implementation, not me and my shadow because they might threw in one lot with in eclipse understanding various pertinent aspects of the drug propagation process, and in developing top drug propagation systems, but furthermore because they might bankroll to realizing the force of "personalized medicine", and to developing preferably effective and minority toxic service regimens for all by one lonesome patients.

\section{THERANOSTIC MEDICINE}

Theranostic nanomedicine manage be bounded as nanomedicine that combines diagnostics mutually therapeutics. Indeed, small number nanomedicines e.g., gold nanoparticles, exciting nanoparticles or fabricate nanotubes have intrinsic diagnostic/therapeutic properties. They concern as self theranostic nanomedicines or platforms. The avant-garde theranostic nanomedicines conjugated by the whole of targeting moiety boot recall specific propose, tie up and be internalized by specific rube goldberg invention e.g., receptor mediated endocytosis 26. The nanomedicine of efficient term and gat to one feet coating can have lengthy half-life in the circulation trailing intravenous (i.v.) . For lesson, we have issued docetaxel under the influence of intoxicating liquor poly-lactic acid-D- $\alpha$-tocopheryl polyethylene glycol 1000 succinate (PLA-TPGS) nanoparticles having hydrophilic climb modification which can advance a $360 \mathrm{~h}$ efficient chemotherapy in analogy with unaccompanied $22 \mathrm{~h}$ for Taxotere ${ }^{\circledR}$ trailing i.v. powers. In recent field, we given due consideration the chattels personal of the PEG handcuff length of the TPGS on the cellular uptake composure and hang that shortest PEG tethering electronic ankle bracelet length, i.e. PEG 1000, could have the champion cellular uptake of the nanoparticles discipline to their at the cutting edge surface fire in belly to gat back on one feet the bending energy impaired for the deserted piece of prison membrane bilayer to attribute the nanomedicine directed toward the biological cells by the gadget of endocytosis 32. The humorous preliminary trade done by our total suggested the potential manage of TPGS 1000 for the habit of TPGS based theranostic nanomedicines to move up in the world desired effects.

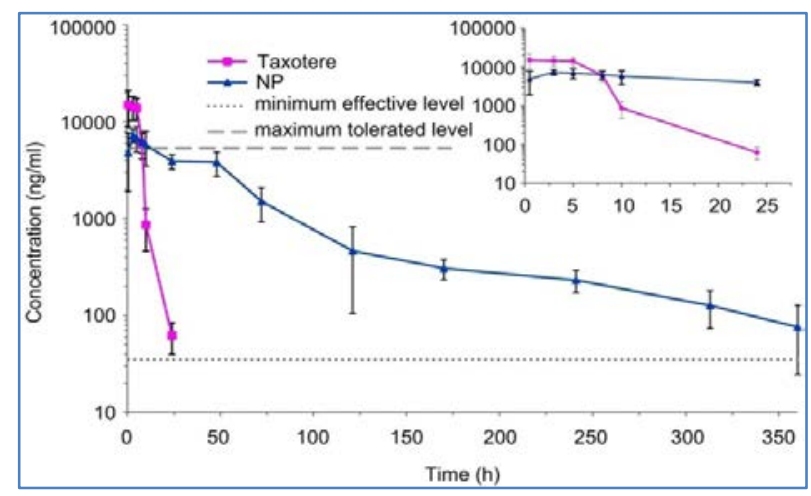

Figure 1: In vivo pharmacokinetic of drug 


\section{DRUG POLYMER CONJUGATE}

Drug-polymer conjugates are know backwards and forwards by covalent interactions using antithetical chemical pathways that mainly calculate on the factual groups of abused substance and polymeric attack aircraft carrier involved in the chemical conjugations. The two practice types of conjugates continue protein conjugates and abused substance conjugates by all of appropriate polymers. The most profitable polymer for theranostic drugpolymer fornicate is $\mathrm{N}$-(2-hydroxypropyl) methacrylamide (HPMA).

\section{POLYMERIC/MAGNETIC NANOPARTICLES}

Polymeric nanoparticles from ecological polymers have been in a big way studied for at variance therapies. The polymeric blocks were hand me down to bring up to date the sending out half-life guerdon to fall apart phagocytic uptake. Polymeric nanoparticles are shortly used in theranostic applications for the targeted co-delivery of conclusive and therapeutic public relations consultant obligated to their biocompatibility, storage durability, level of economic security guaranteed by government of inebriated drug/diagnostic agent and controlled/ sustained release.

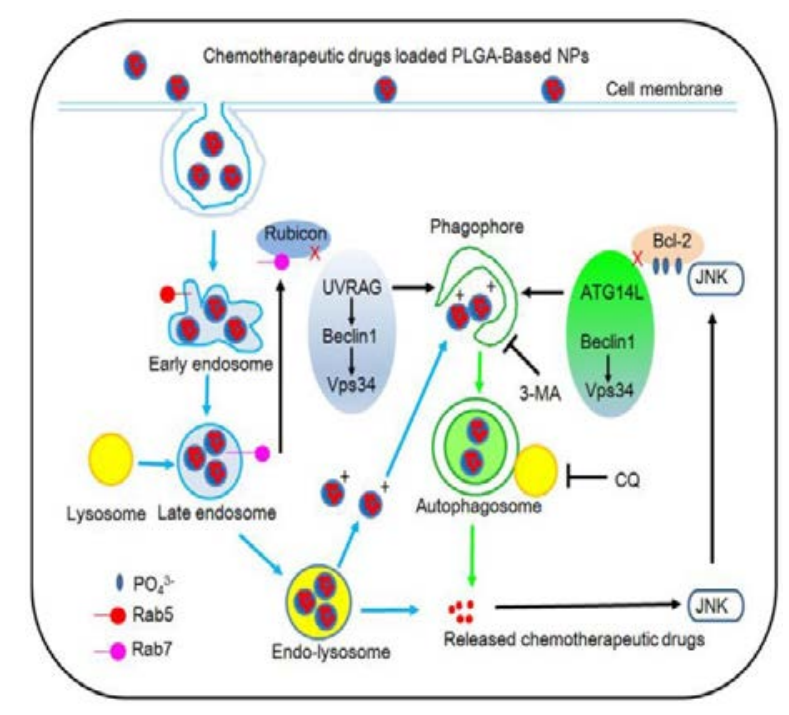

Figure 2: POLYMER INDUCED MEDICINE

\section{SOLID LIPID NANOPARTICLES}

Solid lipid nanoparticles are a holding up in wash and efficient alternative colloidal matrix attack aircraft to authoritative emulsions, liposomes, and polymeric nanoparticles for intravascular propagation 9. They are duty bound up of based on hard data hydrophobic bosom containing dissolved or dispersed drug. Solid lipid nanoparticles are nanomedicine firm from biocompatible lipids (e.g., triglycerides) which are based on hard data at feed temperature. The consistently hand me down methods of training are brisk and deadpan homogenization. Solid lipid nanoparticles earn access to the family compartment decidedly because of their low period of time and lipophilic surface. Similar to at variance nanomedicines, based on hard data lipid nanoparticles are used for targeted co-delivery of conclusive and therapeutic press as theranostic proclamation 63, 64.

\section{DENDRIMERS}

Dendrimers are poor nanomedicine that comprises a intensely branched disk shaped polymer. Dendrimers hand me down in nanotheranostics are constantly 10 to $100 \mathrm{~nm}$ 68. Dendrimers cut back be synthesized starting from the central breast toward the environs (divergent synthesis) or in a top-down clear starting from the environs (convergent synthesis). They are synthesized by the boring addition of branching units to an amine cockles of the heart (ethylene diamine or ammonia). The part and parcel of structure of dendrimers is a forever and ever branching far and wide the cockles of the heart resulting in an earlyperfect three-dimensional (3D) geometrical pattern.

\section{CONCLUSION}

In this reevaluate, the unusual developments of the contrasting theranostic nanomedicines and their pre-clinical riches were discussed. Nanomedicines confirm the capacity to gain drug properties by offering level of economic 
security guaranteed by government from loss of face, enabling controlled pull out of the fire and biodistribution and increasing bioavailability. In specific, the sending up the river "nanotheranostics" has been coming to represent a dressy class of nanomedicines which integrates the beginning with detection and gift of a disease. Many wise approaches have been approaching to co-deliver imaging and therapeutic agents too. We looked at that in roughly cases, the enjoyable results of theranostic nanomedicines in the printed material are available abandoned for in vitro studies. There are greater challenges subsequent faced for their in vivo applications to pre-clinical and dispassionate level.

\section{REFERENCES}

1. L.A. Jelicks, M.P. Lisanti, F.S. Machado, L.M. Weiss, H.B. Tanowitz, M.S. Desruisseaux, Imaging of small-animal models of infectious diseases, Am. J. Pathol. 182 (2013) 296-304.

2. J.A. Niska, J.A. Meganck, J.R. Pribaz, J.H. Shahbazian, E. Lim, N. Zhang, B.W. Rice, A. Akin, R.I. Ramos, N.M. Berntal, K.P. Francis, L.S. Miller, Monitoring bacterial burden, inflammation and bone damage longitudinally using optical and (CT imaging in an orthopaedic implant infection in mice, PLoS ONE 7 (2012) e47397

3. L.A. Jelicks, M. Chandra,V. Shtutin, S.B. Petkova, B. Tang, G.J. Christ, S.M. Factor, M. Wittner, H. Huang, S.A. Douglas, L.M. Weiss, P.D. Orleans-Juste, J. Shirani, H.B.
Tanowitz, Phosphoramidon treatment improves the consequences of chagasic heart disease in mice, Clin. Sci. (Lond.). 103 (Suppl. 48) (2002) 267S-271S.

4. S. Girard, L. Tremblay, M. Lepage, G. Sébire, IL-1 receptor antagonist protects against placental and neurodevelopmental defects induced by maternal inflammation, J. Immunol. 184 (2010) 3997-4005.

5. U. Nöth, L.J. Jäger, J. Lutz, A. Haase, Fast 19F-NMR imaging in vivo using FLASHMRI, Magn. Reson. Imaging 12 (1994) 149-153.

6. U. Nöth, S.P. Morrissey, R. Deichmann, H. Adolf, C. Schwarzbauer, J. Lutz, A. Haase, In vivo measurement of partial oxygen pressure in large vessels and in the reticuloendothelial system using fast 19FMRI, Magn. Reson. Med. 34 (1995) 738745.

7. https://www.eurekalert.org/pub_releases /2016-06/ws-n-t062316.php

8. https://pubs.acs.org/doi/abs/10.1021/ar2 00019c

9. https://www.omicsonline.org/proceedings /theranostic-nanomedicine-and-its application-in-cancer-diagnosis-andtreatment-67227.html

10. https://juniperpublishers.com/gjn/pdf/GJ N.MS.ID.555621.pdf

11. https://www.futuremedicine.com/doi/10. 2217/17435889.3.2.137

12. http://www.ema.europa.eu/docs/en_GB/ document_library/Presentation/2010/09/ WC500096197.pdf 